Nonl. Analysis and Differential Equations, Vol. 1, 2013, no. 2, 83 - 92 HIKARI Ltd, www.m-hikari.com

\title{
An Estimation from within of the Reachable Set of Generalized Nonlinear R. Brockett Integrator with Small Vector Nonlinearity
}

\author{
Aboubacar MOUSSA \\ Department of Mathematics and Computer Science \\ Faculty of Sciences, Abdou Moumouni University \\ BP: 10662 Niamey, Niger \\ msaboubacar@yahoo.fr \\ Adamou OUSMANE MANGA \\ Department of Physics \\ Faculty of Sciences, Abdou Moumouni University \\ BP: 10662 Niamey, Niger \\ manga_adamou@yahoo.com \\ Copyright (c) 2013 Aboubacar MOUSSA and Adamou OUSMANE MANGA. This is an \\ open access article distributed under the Creative Commons Attribution License, which per- \\ mits unrestricted use, distribution, and reproduction in any medium, provided the original
} work is properly cited.

\begin{abstract}
In this paper the generalized nonlinear R. Brockett integrator with small vector nonlinear addition to the rigth-hand side of the corresponding differential equations is considered. More precisely, we investigate the possibility to estimate from within the corresponding reachable set. We obtain some ellipsoidal estimation from within in an efficient form.
\end{abstract}

Mathematics Subject Classification: 49J10

Keywords: Control problem, reachable set, generalized R. Brockett integrator, estimation from within 


\section{Introduction}

One of the fundamental problems in the Control Theory of dynamic systems is the problem of estimation of reachable sets. Reachable sets of controlled objets are the subject of many studies in Mathematical Control Theory. They are studied from different points of view, for example, estimate from above, approximate in the sense of the Hausdorff metric simply constructed convex compact,...

In this paper, we obtain a non-trivial estimation from within for the reachable set of the generalized nonlinear R. Brockett integrator with small vector nonlinearity. Such estimations are object of interest for the Optimal Control Theory and its Applications.

\section{Problem formulation}

We consider the generalized nonlinear R. Brockett integrator with small vector nonlinearity of the form

$$
\begin{aligned}
& \dot{x}_{1}=u_{1}+\varepsilon g_{1}(x, u) \\
& \dot{x}_{2}=u_{2}+\varepsilon g_{2}(x, u) \\
& \dot{x}_{3}=u_{2} x_{1}-u_{1} x_{2}+\varepsilon g_{3}(x, u)
\end{aligned}
$$

with zero initial condition

$$
x(0)=0 .
$$

Where $x \in \mathbf{R}^{3}, \varepsilon \geqslant 0, g_{i}(x, u), \quad i=1,2,3$ are continuous functions on $\mathbf{R}^{3} \times U$. We impose on the control vector $u$ the constrain

$$
u \in U=\left\{u \in \mathbf{R}^{2}:\left|u_{i}\right| \leqslant 1, i=1,2\right\} .
$$

We assume that:

1. For $x \in \mathbf{R}^{3}, u \in U$ is realized the inequality

$$
\langle x, g(x, u)\rangle \leqslant c\left(1+|x|^{2}\right),
$$

where $c>0$ is a constant, $|x|$ means the norm of the vector $x,\langle\cdot, \cdot\rangle$ means the scalar product of vectors and $g(x, u)$ is a continuous vector function with components $g_{i}, \quad i=1,2,3$.

2. The functions $g_{i}(x, u), \quad i=1,2,3$ satisfy the local Lipschitz condition in the variables $(x, u)$ on $\mathbf{R}^{3} \times U$ and

$$
g_{i}(x, u) \not \equiv 0, \quad i=1,2,3 .
$$

Note that if $\varepsilon=0$ the system (1) describes the dynamics of R. Brockett integrator (see [1]- [3]). 
Further, we fix the constant $T>0$. We consider all possible measurable controls $u(t) \in U, t \in \Delta$, where $\Delta=[0, T]$, and the corresponding absolutely continuous solutions $x(t, u(\cdot), \varepsilon)$ of the system (1) with the initial condition (2). The reachable set we are interested in is

$$
D(T, \varepsilon)=\bigcup_{u(\cdot)} x(T, u(\cdot), \varepsilon),
$$

where $u(\cdot)$ is an arbitrary measurable control, satisfying (3) for $t \in \Delta$. From the general theory of nonlinear controlled objects (see, for example, [4, p. 264]), it follows that $\overline{D(T, \varepsilon)}$ is a compact set in $\mathbf{R}^{3}$, where line means the closing of the set. Our objective is to construct for $D(T, \varepsilon)$ such a convex compact set $K \subset \mathbf{R}^{3}$, so that for sufficiently small $\varepsilon \geqslant 0$

$$
K \subset D(T, \varepsilon) \quad \text { and } \quad 0 \in \operatorname{Int} K
$$

where Int $K$ means the interior of the set $K$. We denote by $L_{\infty}(\Delta)$ (see $[5$, p. 31]) the Banach space of 2-dimensional measurable functions $u(t)$ on the interval $\Delta=[0, T]$, substantially bounded in modulus, i. e. vraisup $|u(t)|<$ $+\infty$, and with the usual identification of functions, which coincide almost everywhere on $\Delta$. We define the norm of the element $u(\cdot) \in L_{\infty}(\Delta)$ by the formula

$$
\|u(\cdot)\|=\underset{t \in \Delta}{\operatorname{vrai} \sup }|u(t)| .
$$

For any measurable functions $u(t) \in U, t \in \Delta$ (see (3)) the corresponding solution $x(t, u(\cdot), \varepsilon)$ is uniquely defined with the initial condition (2). Note that it is possible to consider the admissible controls as the elements of $L_{\infty}(\Delta)$.

We will estimate the reachable set $D(T, \varepsilon)$ of the system (1), (2) from within.

Note that when $\varepsilon=0$ the corresponding estimate is obtained in the work [3] of M. S. Nikolskii.

\section{Solution of problem}

These are the main results of the paper. We denote by $y(T, u(\cdot))$ the solution of R. Brockett system of equations (see [1]-[3])

$$
\begin{aligned}
& \dot{y}_{1}=u_{1} \\
& \dot{y}_{2}=u_{2} \\
& \dot{y}_{3}=u_{2} y_{1}-u_{1} y_{2}
\end{aligned}
$$

where $y(0)=0$ and the admissible $u(t) \in U, t \in \Delta$. 
Then it is easy to show that the solution $x(T, u(\cdot), \varepsilon)$ of the system (1), (2) allows the following representation for admissible $u(t) \in U, t \in \Delta$ :

$$
x(T, u(\cdot), \varepsilon)=y(T, u(\cdot))+\varepsilon R(u(\cdot), \varepsilon),
$$

where

$$
y(T, u(\cdot))=A u(\cdot)+\frac{1}{2} C[u(\cdot), u(\cdot)],
$$

and $A$ is a linear vector-bounded operator, acting from $L_{\infty}(\Delta)$ to $\mathbf{R}^{3}$ by the formulas for its component of kind

$$
A_{i} u(\cdot)=\int_{0}^{T} u_{i}(s) d s, \quad i=1,2 ; \quad A_{3} u(\cdot)=0
$$

$C[u(\cdot), u(\cdot)]$ is a continuous vector quadratic form (see [5], [6]) with components

$$
\begin{gathered}
C_{i}[u(\cdot), u(\cdot)]=0, \quad i=1,2, \\
C_{3}[u(\cdot), u(\cdot)]=2 \int_{0}^{T}\left(u_{2}(s) y_{1}(s, u(\cdot))-u_{1}(s) y_{2}(s, u(\cdot))\right) d s,
\end{gathered}
$$

here (see (8))

$$
y_{i}(t, u(\cdot))=\int_{0}^{t} u_{i}(s) d s, \quad i=1,2,
$$

$R(u(\cdot), \varepsilon)$ is a nonlinear vector function, and also (see (1), (5))

$$
R(u(\cdot), \varepsilon)=\int_{0}^{T} g(x(s, u(\cdot), \varepsilon), u(s)) d s
$$

Using the inequality (4) and the results of [7] we can show that the function $|R(u(\cdot), \varepsilon)|$ is bounded for $\varepsilon \in[0,1]$ and any admissible $u(t) \in U, t \in \Delta$, i. e.

$$
|R(u(\cdot), \varepsilon)| \leqslant k_{1}
$$

where

$$
k_{1}=c T e^{(2+c) T}
$$

Using the local Lipschitz condition in $(x, u)$ of the functions $g_{i}(x, u), \quad i=$ $1,2,3$ on $\mathbf{R}^{3} \times U$, the inequality (16), we can prove the continuity in $(u(\cdot), \varepsilon)$ of the nonlinear mapping $R(u(\cdot), \varepsilon)$ on the set of all admissible controls $u(\cdot)$, $\varepsilon \in[0,1]$ in the norm of $L_{\infty}(\Delta) \times[0,1]$. From formula (11) we see that, 
$A L_{\infty} \neq \mathbf{R}^{3}$, i. e. the linear operator does not realize a covering of the space $\mathbf{R}^{3}$.

We consider the admissible control $\tilde{u}(t) \in U, t \in \Delta$, with the components

$$
\tilde{u}_{i}(t)=\alpha(t), \quad i=1,2,
$$

where

$$
\alpha(t)=\frac{1}{2} \quad \text { for } \quad t \in\left[0, \frac{T}{2}\right], \quad \alpha(t)=-\frac{1}{2} \quad \text { for } \quad t \in\left(\frac{T}{2}, T\right] .
$$

It is easy to see that (see $(11)-(14))$ for $\tilde{u}(t), t \in \Delta$, and its corresponding solution $\tilde{x}(t)$ of system $(1),(2)$ the equalities

$$
A \tilde{u}(\cdot)=0, \quad C[\tilde{u}(\cdot), \tilde{u}(\cdot)]=0, \quad \tilde{x}(T)=\varepsilon R(\tilde{u}(\cdot), \varepsilon)
$$

are realized. We consider for $t \in \Delta$ and $\mu \in(0,1]$ the admissible control

$$
u_{\mu}(t)=\mu \tilde{u}(t)+\mu^{3 / 2} \nu(t),
$$

where the measurable function $\nu(t) \in \mathbf{R}^{2}$ satisfies on $\Delta$ the inequality

$$
|\nu(t)| \leqslant \frac{1}{2}
$$

From (9), (10), (19) and (20) we obtain the following formula for the solution $x_{\mu}(t)$ of the system $(1),(2)$, that corresponding to the control $u_{\mu}(\cdot)$ :

$x_{\mu}\left(T, u_{\mu}(\cdot), \varepsilon\right)=\mu^{3 / 2} A \nu(\cdot)+\mu^{5 / 2} C[\tilde{u}(\cdot), \nu(\cdot)]+\frac{\mu^{3}}{2} C[\nu(\cdot), \nu(\cdot)]+\varepsilon R\left(u_{\mu}(\cdot), \varepsilon\right)$,

where $C[\xi, \eta]$ is a continuous symmetric bilinear form (here $\xi, \eta$ are arbitrary elements of $L_{\infty}(\Delta)$ ). We note that (see (12)-(14), (17)-(19))

$$
C_{i}[\xi, \eta]=0, \quad i=1,2
$$

for arbitrary $\xi, \eta$ from $L_{\infty}(\Delta)$ and

$$
C_{3}[\tilde{u}(\cdot), \nu(\cdot)]=\int_{0}^{T}\left[\alpha(s) \int_{0}^{s}\left(\nu_{1}(r)-\nu_{2}(r)\right) d r-\int_{0}^{s} \alpha(r) d r\left(\nu_{1}(s)-\nu_{2}(s)\right)\right] d s,
$$

where $\nu_{i}(t), i=1,2$, are the components of the vector function $\nu(t)$. Further (see (18)), $\int_{0}^{T} \alpha(\tau) d \tau=0$, therefore

$$
\int_{r}^{T} \alpha(\tau) d \tau=-\int_{0}^{r} \alpha(\tau) d \tau
$$


for $r \in \Delta$.

Changing the order of integration in the first iterated integral (24) and using the formula (25), we can rewrite (24) in the following form:

$$
C_{3}[\tilde{u}(\cdot), \nu(\cdot)]=-2 \int_{0}^{T}\left[\int_{0}^{s} \alpha(r) d r\left(\nu_{1}(s)-\nu_{2}(s)\right)\right] d s .
$$

We consider now the linear operator $N$, acting from $L_{\infty}(\Delta)$ to $\mathbf{R}^{3}$ by the formula

$$
N \nu(\cdot)=A \nu(\cdot)+C[\tilde{u}(\cdot), \nu(\cdot)]
$$

We note that the linear operator $N$ is closely connected with the formula (22). From the formulas (11), (23), (24) it follows that

$$
\begin{gathered}
N_{i} \nu(\cdot)=\int_{0}^{T} \nu_{i}(s) d s, \quad i=1,2, \\
N_{3} \nu(\cdot)=C_{3}[\tilde{u}(\cdot), \nu(\cdot)],
\end{gathered}
$$

where $N_{i} \nu(\cdot)$ is a $i$-th component of the vector $N \nu(\cdot)$.

We denote by $W$ the set of functions $\nu(\cdot) \in L_{\infty}(\Delta)$, which are almost everywhere refer to the zero vector for $t \in\left[\frac{T}{2}, T\right]$. On the set $W$ (see (12), (17), (18), (26)-(29))

$$
\begin{aligned}
& N_{i} \nu(\cdot)=\int_{0}^{T / 2} \nu_{i}(s) d s, \quad i=1,2, \\
& N_{3} \nu(\cdot)=-\int_{0}^{T / 2} s\left(\nu_{1}(s)-\nu_{2}(s)\right) d s .
\end{aligned}
$$

From formulas (30), (31) it follows that on $W$

$$
N \nu(\cdot)=\int_{0}^{T / 2} B(s) \nu(s) d s
$$

where

$$
B(s)=\left(\begin{array}{rr}
1 & 0 \\
0 & 1 \\
-s & s
\end{array}\right)
$$


We fix an arbitrary vector $\varphi \in \mathbf{R}^{3}$ and consider for $s \in[0, T / 2]$ the function

$$
\nu_{\varphi}(s)=B^{*}(s) \varphi
$$

where $*$ denotes the transpose of a matrix. Assuming $\nu_{\varphi}(s)=0$ for $s \in$ $\left(\frac{T}{2}, T\right]$, from $(32),(34)$, we obtain that

$$
N \nu_{\varphi}(\cdot)=G \varphi
$$

where

$$
G=\int_{0}^{T / 2} B(s) B^{*}(s) d s,
$$

$\varphi$-an arbitrary vector from $\mathbf{R}^{3}$. From the formulas (32), (35) we obtain that

$$
G=\left(\begin{array}{ccc}
\beta & 0 & -\beta^{2} / 2 \\
0 & \beta & \beta^{2} / 2 \\
-\beta^{2} / 2 & \beta^{2} / 2 & \frac{2}{3} \beta^{3}
\end{array}\right),
$$

where $\beta=\frac{T}{2}$. Hence, the matrix $G$ is nondegenerate and

$$
N W=\mathbf{R}^{3}, \quad N L_{\infty}(\Delta)=\mathbf{R}^{3} .
$$

From formulas (26)-(29) it follows that on the functions $\nu(\cdot) \in L_{\infty}(\Delta)$ the linear operator $N$ is described by the following formula (cf. (32), (33)):

$$
N \nu(\cdot)=\int_{0}^{T} B(s) \nu(s) d s,
$$

where for $s \in \Delta$

$$
B(s)=\left(\begin{array}{c}
1,0 \\
0,1 \\
-2 \int_{0}^{s} \alpha(r) d r, 2 \int_{0}^{s} \alpha(r) d r
\end{array}\right)
$$

We consider now for $s \in \Delta$ the multivalued mapping

$$
\Omega(s)=B(s) \sigma,
$$

where $\sigma=\left\{\nu \in \mathbf{R}^{2}:|\nu| \leqslant \frac{1}{2}\right\}$, and its integral (see [5], [8])

$$
P=\int_{0}^{T} \Omega(s) d s .
$$


On the basis of the general theory of multivalued maps (see [5], [8]) and formula (38), we have

$$
P=N V
$$

where $V$ is the set of measurable functions on $\Delta$ satisfying the inequality (21). Next, using the theory of multivalued maps it is proved that $P$ is the convex compact set containing the point 0 . With the help of (37) it is easy to show that

$$
0 \in \operatorname{Int} P \text {. }
$$

Using the formulas (11), (12), (23), (27)-(29) we rewrite the formula (22) in the form

$$
x_{\mu}\left(T, u_{\mu}(\cdot), \varepsilon\right)=\Lambda(\mu)\left(N \nu(\cdot)+\frac{\mu^{1 / 2}}{2} C[\nu(\cdot), \nu(\cdot)]+\varepsilon \Lambda^{-1}(\mu) R\left(u_{\mu}(\cdot), \varepsilon\right)\right),
$$

where

$$
\Lambda(\mu)=\left(\begin{array}{ccc}
\mu^{3 / 2} & 0 & 0 \\
0 & \mu^{3 / 2} & 0 \\
0 & 0 & \mu^{5 / 2}
\end{array}\right)
$$

Recall that in (43), $\nu(t)$ is an arbitrary mesurable function on $\Delta$, satisfying there the inequality $(21)$.

For the vector quadratic form $C[\nu(\cdot), \nu(\cdot)]$ (see (12)-(14)) it is easy to prove the uniform estimation for all admissible $\nu(\cdot)$

$$
|C[\nu(\cdot), \nu(\cdot)]| \leqslant \frac{1}{2} \int_{0}^{T} s d s=\frac{T^{2}}{4} .
$$

Let us denote

$$
\eta=\max _{s \in[0, T / 2]}\left\|B^{*}(s)\right\|
$$

where

$$
\left\|B^{*}(s)\right\|=\max _{|y| \leqslant 1}\left|B^{*}(s) y\right|, \quad y \in \mathbf{R}^{3} .
$$

It is clearly that $\eta>0$ and is easy to see that the continuous function $\nu_{\varphi}(s)$ (see $(34)$ ) for $s \in[0, T / 2]$ and $|\varphi| \leqslant \frac{1}{2 \eta}$ satisfies the condition

$$
\left|\nu_{\varphi}(s)\right| \leqslant \frac{1}{2}
$$

Assuming $\nu_{\varphi}(s)=0$ for $s \in\left(\frac{T}{2}, T\right]$, with the help of formulas (34)-(36), (39), (40), (46) and the definition of the integral of multivalued mapping (see, for 
example [5], [8]) it is easy to justify the inclusion

$$
\frac{1}{2 \eta} G S \subset P
$$

where

$$
S=\left\{y \in \mathbf{R}^{3}:|y| \leqslant 1\right\} .
$$

Below we will show that the set $G S$ with an appropriate matrix mutiplier determines an estimation from within of $D(T, \varepsilon)$ for sufficiently small $\varepsilon \geqslant 0$. We introduce (see (43), (44)) the map

$$
F(\nu(\cdot), \mu, \varepsilon)=N \nu(\cdot)+\frac{\mu^{1 / 2}}{2} C[\nu(\cdot), \nu(\cdot)]+\varepsilon R_{1}(\nu(\cdot), \mu, \varepsilon),
$$

where $\nu(\cdot)$ is an arbitrary measurable function satisfying the inequality (21),

$$
\mu \in(0,1], \quad \varepsilon \in[0,1] \quad \text { and } \quad R_{1}(\nu(\cdot), \mu, \varepsilon)=\Lambda^{-1}(\mu) R\left(u_{\mu}(\cdot), \varepsilon\right) .
$$

We denote $($ see $(34)) F_{1}(\varphi, \mu, \varepsilon)=F\left(\nu_{\varphi}(\cdot), \mu, \varepsilon\right)$, where

$$
\varphi \in \frac{1}{2 \eta} S, \quad S=\left\{y \in \mathbf{R}^{3}:|y| \leqslant 1\right\}, \quad \mu \in(0,1] \quad \text { and } \quad \varepsilon \in[0,1] .
$$

Now we obtain the representation

$$
F_{1}(\varphi, \mu, \varepsilon)=G\left(\varphi+\frac{\mu^{1 / 2}}{2} G^{-1} C\left[\nu_{\varphi}(\cdot), \nu_{\varphi}(\cdot)\right]+\varepsilon G^{-1} R_{1}\left(\nu_{\varphi}(\cdot), \mu, \varepsilon\right)\right),
$$

where $\varphi \in \frac{1}{2 \eta} S, \quad \mu \in(0,1]$ and $\varepsilon \in[0,1]$. We denote

$$
\left.F_{2}(\varphi, \mu, \varepsilon)=\varphi+\frac{\mu^{1 / 2}}{2} G^{-1} C\left[\nu_{\varphi}(\cdot)\right], \nu_{\varphi}(\cdot)\right]+\varepsilon G^{-1} R_{1}\left(\nu_{\varphi}(\cdot), \mu, \varepsilon\right),
$$

where $\varphi \in \frac{1}{2 \eta} S, \quad \mu \in(0,1]$ and $\varepsilon \in[0,1]$. Fix $\mu_{0} \in(0,1]$ such that (see (45), (49), (50))

$$
\frac{\mu_{0}^{1 / 2}}{2}\left\|G^{-1}\right\| \frac{T^{2}}{4} \leqslant \frac{1}{6 \eta}
$$

Fix $\varepsilon_{0} \in(0,1]$, such that (see (16), (44), (47)-(50))

$$
\varepsilon_{0}\left\|G^{-1}\right\| \mu_{0}^{-5 / 2} k_{1} \leqslant \frac{1}{6 \eta}
$$

Then (see the Corollary of the Topological Theorem in [4], p. 276, 277) for $\varepsilon \in\left[0, \varepsilon_{0}\right]$ we obtain the inclusion

$$
F_{2}\left(\frac{1}{2 \eta} S, \mu_{0}, \varepsilon_{0}\right) \supset \frac{1}{6 \eta} S .
$$


From the above, it follows that, (see (43), (49), (50)) for $\varepsilon \in\left[0, \varepsilon_{0}\right]$ the required inclusion

$$
\frac{1}{6 \eta} \Lambda\left(\mu_{0}\right) G S \subset D(T, \varepsilon)
$$

is realized.

ACKNOWLEDGEMENTS. The authors are grateful to Professor M. S. Nikolskii, V.A. Steklov Mathematical Institute of the Russian Academy of Sciences for helpful discussions, encouragement and reference [3].

\section{References}

[1] R.W. Brockett, Asymptotic stability and feedback stabilization, Differential Geometric Control Theory, Proceedings of Conference of the Michigan Technological University, 1982, 181-191.

[2] M. Kavski, Chronological algebras, Results of Science and Technology, Contemporary Mathematics and its applications, Thematic reviews, 60 VINITI, Moscow, 1999, 144 - 178.

[3] M. S. Nikolskii, About an estimate of reachable set of R. Brockett nonlinear integrator, Differential Equations, 36, (11) 2000, 1501-1505. (in Russian)

[4] E.B. Lee and L. Markus, Foundations of the Optimal Control Theory, John Wiley \& Sons, New York, 1970. (in Russian)

[5] A.D. Ioffe and V.M. Tikhomirov, Theory of extremal problems, Nauka, Moscow., 1974. (in Russian)

[6] V.M. Alekseev, V.M. Tikhomirov and S.V. Fomin, Optimal Control, Nauka, Moscow, 1979. (in Russian)

[7] A.F. Filippov, On some questions of optimal regulation, Vestnik of Moscow University, Mathematics, Mechanics, Astronomy, Physics, Chemistry, 2 (1959) 25-32. (in Russian)

[8] V.I. Blagodatskikh and A.F. Filippov, Differential Inclusions and Optimal Control, Proceedings of Steklov Mathematical Institute of the USSR Academy of Sciences, 169 (1985) 194-252. (in Russian)

\section{Received: February 10, 2013}

